\title{
Contribution of the Circadian Pacemaker and the Sleep Homeostat to Sleep Propensity, Sleep Structure, Electroencephalographic Slow Waves, and Sleep Spindle Activity in Humans
}

\author{
Derk-Jan Dijk and Charles A. Czeisler \\ Section on Sleep Disorders/Circadian Medicine, Division of Endocrinology, Department of Medicine, Harvard \\ Medical School and Brigham and Women's Hospital, Boston, Massachusetts 02115
}

The role of the endogenous circadian pacemaker in the timing of the sleep-wake cycle and the regulation of the internal structure of sleep, including REM sleep, EEG slowwave $(0.75-4.5 \mathrm{~Hz})$ and sleep spindle activity (12.75-15.0 $\mathrm{Hz}$ ) was investigated. Eight men lived in an environment free of time cues for 33-36 d and were scheduled to a 28 $\mathrm{hr}$ rest-activity cycle so that sleep episodes ( $9.33 \mathrm{hr}$ each) occurred at all phases of the endogenous circadian cycle and variations in wakefulness preceding sleep were minimized. The crest of the robust circadian rhythm of REM sleep, which was observed throughout the sleep episode, was positioned shortly after the minimum of the core body temperature rhythm. Furthermore, a sleep-dependent increase of REM sleep was present, which, interacting with the circadian modulation, resulted in highest values of REM sleep when the end of scheduled sleep episodes coincided with habitual wake-time. Slow-wave activity decreased and sleep spindle activity increased in the course of all sleep episodes. Slow-wave activity in non-REM sleep exhibited a low amplitude circadian modulation which did not parallel the circadian rhythm of sleep propensity. Sleep spindle activity showed a marked endogenous circadian rhythm; its crest coincident with the beginning of the habitual sleep episode. Analyses of the (nonadditive) interaction of the circadian and sleep-dependent components of sleep propensity and sleep structure revealed that the phase relation between the sleep-wake cycle and the circadian pacemaker during entrainment promotes the consolidation of sleep and wakefulness and facilitates the transitions between these vigilance states.

[Key words: sleep, circadian rhythm, REM sleep, nonREM sleep, slow wave sleep, slow-wave activity, sleep spindle, electroencephalogram, sleep propensity, body temperature, homeostasis, suprachiasmatic nuclei]

\footnotetext{
Received July 20, 1994; revised Nov. 17, 1994; accepted Nov. 29, 1994.

We thank Allan E. Ward and David W. Rimmer for supervising the studies, the research and student technicians for data collection, Joseph $M$. Ronda for programming assistance, J. Boyd Hayes and Walter O. Freitag for technical support, Jeanne F. Duffy for her advice, and Drs. Richard E. Kronauer, Steven H. Strogatz, Emery N. Brown, Elizabeth B. Klerman, Frederick C. Davis, Joanne Waldstreicher, and Gordon H. Williams for their thoughtful discussions. This work was supported by USPHS NIH Awards NIA-1-P01-AG09975, NCRRGCRC-M01-RR02635, and NIMH-1-R01-MH45130.

Correspondence should be addressed to Dr. Derk-Jan Dijk, Institute of Pharmacology, University of Zürich, Winterthurerstrasse 190, Cll 8057, Zürich, Switzerland.

Copyright (C) 1995 Society for Neuroscience $0270-6474 / 95 / 153526-13 \$ 05.00 / 0$
}

The suprachiasmatic nuclei ( $\mathrm{SCN}$ ) of the hypothalamus are the locus of an endogenous self-sustaining circadian pacemaker (Moore and Eichler, 1972; Stephan and Zucker, 1972; for reviews, see Rusak and Zucker, 1979; Meijer and Rietveld, 1989; Klein et al., 1991) regulating circadian rhythms of a variety of physiological variables including plasma melatonin (Moore and Klein, 1974), plasma corticosterone (Moore and Eichler, 1972) and core body temperature (Eastman et al., 1984; Edgar, 1986). The SCN are also involved in the timing of sleep and wakefulness, as has been demonstrated by lesion studies in both rodents (Mistlberger et al., 1983; Tobler et al., 1983; Eastman et al., 1984; Trachsel et al., 1992) and the squirrel monkey (Edgar, 1986; Edgar et al., 1993). In humans, lesions near or in an area homologous to the SCN cause severe disruptions of the sleepwake cycle (Cohen and Albers, 1991), consistent with the suggestion more than a half-century ago that this region of the brain governs the timing of sleep (Fulton and Bailey, 1929).

Despite the progress that has since been made in the understanding of the pacemaker properties of the SCN, the identification of the pathways by which this pacemaker is entrained to the daily light-dark cycle, and the demonstration that light is also the principal synchronizer of this pacemaker in humans (for review, see Czeisler, 1994), it has remained unclear how the outputs of the pacemaker regulate the timing of human bchavior, including sleep (Watts, 1991). The role of the circadian pacemaker in the regulation of human sleep has been investigated in long-term temporal isolation studies, short sleep-wake cycle designs, sleep displacement paradigms, and studies in which the circadian pacemaker was shifted by scheduled exposure to bright light. The classical temporal isolation studies, including those in which the sleep-wake cycle spontaneously desynchronized from the body temperature rhythm, have highlighted the circadian regulation of spontaneous sleep initiation, and sleep duration, and the influence of circadian phase on REM sleep (Aschoff, 1965; Aschoff et al., 1967; Czeisler, 1978; Wever, 1979; Zulley, 1979, 1980; Czeisler et al., 1980a,b; Zulley et al., 1981; Strogatz et al., 1986). Short sleep-wake cycle designs in which subject were scheduled to sleep for one-third of imposed 20,90, 180, or 240 min rest-activity cycles that were then maintained for one to ten $24 \mathrm{hr}$ days, focused primarily on the circadian variation in sleep propensity (Weitzman et al., 1974; Carskadon and Dement, 1975; Webb and Agnew, 1975; Czeisler, 1978; Lavie, 1986; Zulley, 1990). Sleep displacement studies in which sleep was scheduled to daytime hours by varying the duration of wakefulness have emphasized the relation between prior wakefulness and sleep propensity and the internal structure of sleep (Webb and 
Agnew, 1971; Carskadon and Dement, 1979; Åkerstedt and Gillberg, 1981, 1986; Borbély et al., 1981; Dinges, 1986; Dijk et al., 1987a, 1990a,b, 1991; Gillberg and Åkerstedt, 1991). Light exposure studies have investigated the effects of moderate phase shifts of the circadian pacemaker on the timing of awakening, the internal structure of sleep, and the EEG in non-REM sleep (Dijk et al., 1987b, 1989; Drennan et al., 1989; Campbell et al., 1993). Consistent findings across these different protocols are that sleep propensity and REM sleep peak at or shortly after the trough of the endogenous component of the core body temperature rhythm, and that slow wave sleep, that is, stages $3+4$ of non-REM sleep, and EEG power density in non-REM sleep are largely independent from endogenous circadian phase and are primarily determined by the duration of prior wakefulness and sleep. The data summarized above support the concept that the timing of sleep and wakefulness and sleep structure results from the interaction of a circadian and a sleep-wake dependent pro cess (Borbély, 1982; Daan et al., 1984; Beersma et al., 1987; Edgar et al., 1993). However, the strength of the circadian and the homeostatic, that is, the sleep-wake dependent, components modulating sleep propensity and sleep structure and their interaction could not be quantified by any of these protocols. This is because in most of these protocols the phase at which sleep occurred and the duration of prior sleep or wakefulness were confounded (spontaneous desynchrony and displacement of sleep) or the duration of sleep was too short to quantify the sleep dependent component (short sleep-wake cycles). Furthermore, in many of these experiments quantitative EEG analysis was not applied and therefore the influence of the circadian pacemaker on the hallmarks of EEG synchronization during non-REM sleep, that is, electroencephalographic slow waves and activity in the frequency range of sleep spindles (see Steriade et al., $1993 a$ ), remains to be quantified.

As we reported (Dijk and Czeisler, 1994), circadian and sleepdependent components of sleep regulation can be separated by scheduled uncoupling of the rest-activity cycle from the output of the circadian pacemaker in the forced desynchrony protocol, pioneered by Nathaniel Kleitman (1939). Here we present an analysis of the contribution of the circadian pacemaker and the sleep homeostat and of their interaction, in the regulation of the internal structure of sleep, including REM sleep, EEG slow waves, and sleep spindle activity.

\section{Materials and Methods}

Subjects. Eight male subjects (age $21-28$ years) were paid to participate in the study to which they each gave their informed consent. The study was approved by the Committee for the Protection of Human Subjects from Research Risks at the Brigham and Women's Hospital. They subjects had no evidence of medical or psychiatric disorders revealed by history, physical examination, chest radiography, electrocardiography, biochemical screening, and a psychological screening questionnaire (the Minnesota Multiphasic Personality Inventory). Subjects were screened for sleep disturbances by a sleep disorders questionnaire. Prior to the study the subjects were asked to maintain a regular sleep-wake cycle for at least three weeks. This was verified by subjects calling in to a timc-stamped answering machine at their bedtimes and wake-times for 3 weeks, and by ambulatory monitoring of core body temperature, electrocardjogram and wrist activity on a Vitalog recorder during the week prior to study. On their arrival in the laboratory, their habitual bedtime was determined on the basis of a sleep log and the Vitalog recording during the week prior to the study.

Protocol. Subjects lived in the laboratory for 33-36 d. During the first 3 nights (in one subject only 1 night) subjects were scheduled to sleep for $8 \mathrm{hr}$ at their habitual bedtimes. During the baseline days, subjects remained in their suites in which light intensity was approximately 150 lux. Upon awakening from the last baseline night, subjects stayed in bed in a semirecumbent position during a $40 \mathrm{hr}$ constant routine at a light intensity of $\sim 10-15$ lux. During this constant routine (CR) protocol, which allows assessment of the phase of endogenous circadian rhythms in the absence of the masking effects of sleep, meals, etc. (Czeisler et al., 1985), subjects were given hourly snacks, which satisfied their caloric demand, with $\sim 150 \mathrm{mEq}$ of sodium and $\sim 100$ $\mathrm{mEq}$ of potassium evenly distributed over a $24 \mathrm{hr}$ period. A technician attended the subjects during this CR-protocol in order to prevent the subjects from falling asleep. Polysomnographic recordings were also obtained throughout the CR. At the end of the $40 \mathrm{hr}$ CR subjects were scheduled to sleep for $9 \mathrm{hr}: 20 \mathrm{~min}$. Next the subjects were scheduled to a $28 \mathrm{hr}$ day of which $9 \mathrm{hr}: 20 \mathrm{~min}$ were spent in hed in total darkness each cycle. During their scheduled waking day light intensity was kept at low levels $(\sim 10-15$ lux in seven subjects and $-40-60$ lux in one subject). Theoretically this light intensity does not appreciably alter the period of the circadian pacemaker (Kronauer, 1990; Klerman et al., 1992). Technicians who were in contact with the subjects were trained not to convey time of day information and subjects were without access to clocks, nor other knowledge of the time of day. Subjects were given three meals and could read, study, or play games. At the end of the protocol a final CR was carried out followed by a final sleep episode in the laboratory. Throughout the experiment subjects had to fill out visual analog scales and performance tests and, during some portions of the studies, multiple frequent samples of blood were collected from an indwelling intravenous catheter with side port holes (Deseret Medical Inc., Sandy, UT) placed in a forearm vein.

Body temperature recording. Throughout the entire study period core body temperature was recorded at 1 min intervals from a disposable thermistor (Yellow Springs Instrument Co., Yellow Springs, OH) inserted $10 \mathrm{~cm}$ into the rectum. All temperature data were inspected visually and data segments which were affected by removal or malfunctioning of the temperature sensor were removed. If the length of these segments was shorter or equal to $45 \mathrm{~min}$, data were replaced by a value derived from a linear interpolation procedure.

Sleep recording and analysis. During all sleep episodes EEG, EOG, EMG, and ECG were recorded on Nihon Kohden-5208 or 4418 electroencephalngraphs. F.F.Gs were derived from $\mathrm{C} 3-\mathrm{A} 2, \mathrm{C} 4-\mathrm{A} 1$, and $\mathrm{O} 2-$ A1. EEG signals were high pass filtered with a time constant of $0.3 \mathrm{sec}$ and low pass filtered at $35 \mathrm{~Hz}$. In seven subjects, the EEG signals derived from $\mathrm{C} 3-\mathrm{A} 2$ and $\mathrm{C} 4-\mathrm{A} 1$ wore digitizcd at a sampling rate of $128 \mathrm{~Hz}$ by a 12 bit peripheral laboratory accelerator. The data were stored on disk of a Vax 11/750 computer. EEGs from those subjects were subjected to a spectral analysis by an IMSL FFT routine in which a Parzen spectral window was implemented. Power spectra were calculated per $4 \mathrm{sec}$ epochs, which results in a resolution of $0.25 \mathrm{~Hz}$. Data were reduced by collapsing $0.25 \mathrm{~Hz}$ bins into $0.5 \mathrm{~Hz}$ bins, by omitting spectra above $30 \mathrm{~Hz}$, and averaging over seven or eight 4 sec epochs resulting in one spectrum per 28 or $32 \mathrm{sec}$. Here we present data on slow-wave activity, that is, power density in the $0.75-4.5 \mathrm{~Hz}$ range and sigma activity, that is, power density in the $12.75-15.0 \mathrm{~Hz}$, which corresponds closely to sleep spindle activity as assessed by transient pattern recognition software (Dijk et al., 1993). All epochs with artifacts were identified. Power spectra were calibrated by recording a $16 \mathrm{~Hz}, 25 \mu \mathrm{V}$ calibration signal prior to each sleep episode.

Polysomnographic signals (EEG, EOG, EMG, ECG) were also digitized at a sampling rate of $272 \mathrm{~Hz}$ and stored on a Sun 386i work station. On this workstation the signals were analyzed by Nicolet-UltraSom spectral temporal analysis.

All EEGs were scored manually by one of us (D.J.D.) according to established criteria (Rechtschaffen and Kales, 1968) per $30 \mathrm{sec}$ epochs and synchronized with the power spectra.

Fstimation of endogenous circadian period and phase. The main aim of the present analysis was to quantify circadian and sleep dependent aspects of human sleep regulation. To this end we first estimated for cach subject scparatcly, circadian phase for every minute of the forced desynchrony part of the protocol. Circadian period and phase were assessed in three ways. In the first approach the endogenous circadian minimum of the core body temperature rhythm during the initial and final constant routine was assessed by a dual harmonic regression model developed by Brown and Czeisler (1992). For this assessment, the first $5 \mathrm{hr}$ of the constant routine data were excluded from the analysis because these data are still affected by the masking effects of sleep. The difference in the phase of the endogenous circadian temperature minimum from the first to the final constant routine was used to estimate the endogenous circadian period. The second and third method utilized 
the entire data train of core body temperature, starting at the end of the first sleep episode after the initial constant routine and ending at the end of the last sleep episode prior to the final constant routine. In the second method, the body temperature data were subjected to a periodogram analysis called the minimum variance technique (Czeisler, 1978). This method, which does not make any assumptions on the waveform of the rhythm and therefore can be considered a form of nonparametric spectral analysis, determines the residual variance after the data have been averaged at a certain period. By varying the period at which the data are "folded" in I min increments, these periods which explain most of the variance can be identified. In the forced desynchrony protocol, there will always be a $28 \mathrm{hr}$ component due to the masking effects of the imposed activity and sleep schedule on body temperature, but in addition an endogenous component, which lies close to $24 \mathrm{hr}$, can be identified. In the third method, two sinusoids are fitted to the data simultaneously. The period of one of the sinusoids is set to $28 \mathrm{hr}$, which is the period of the imposed sleep-wake cycle. The period of the other sinusoid and the amplitude and phase of both sinusoids are varied based on a least squares error criterion. This method, developed by Kronauer (Lee et al., 1992), has been called a nonorthogonal spectral analysis. The underlying assumption in all three methods is that the period of the endogenous circadian pacemaker is constant throughout the protocol. This has been validated by simultaneous assessment of period and phase of other variables including plasma melatonin (Shanahan and Czeisler, 1991a) and cortisol at regular intervals throughout the protocol.

The estimates of the endogenous circadian period obtained by all three methods were very similar and the largest difference observed between any of the methods was $9 \mathrm{~min}$. We decided to use the estimate obtained by the nonparametric spectral analysis for the assignment of circadian phase because (1) it does not make any assumptions regarding the waveform of the endogenous component of the body temperature rhythm and (2) the estimate is based on a large portion of the available data and is therefore likely to be quite robust.

For the analysis of the sleep data only the sleep episodes during the forced desynchrony part of the protocol were used. The sleep episodes after the constant routine were excluded from the analyses because of the potential confounding effects of the sleep deprivation inherent to the CR protocol. To separate the circadian and sleep-wake dependent components of sleep propensity and sleep structure, the data were either folded at the endogenous circadian period or at 28 hr. This was achieved by computing for all $30 \mathrm{sec}$ EEG epochs circadian phase and the time elapsed since the start of the sleep episode. The circadian component was computed by averaging the data with respect to circadian phase, irrespective of the time elapsed since the start of the sleep episode. The sleep dependent component was computed by averaging the data with respect to the time elapsed since start of sleep episode, irrespective of circadian phase. The implicit assumption of this method is that the number of sleep episodes $(n=174)$ is large enough and the distribution of these sleep episodes over the circadian cycle is sufficiently uniform that folding the data at the endogenous circadian period will, on average, extract the circadian component and minimize the effect of sleep dependent changes on the educed wave-form; similarly, folding the data at $28 \mathrm{hr}$ will, on average, extract the sleep-wake dependent components and minimize the circadian influences on the educed wave-form.

The interaction between circadian and sleep dependent components was analyzed by computing the circadian modulation as a function of lime elapsed since the stant of the sleep episode and by computing the sleep dependent changes as a function of circadian phase.

Statistics. Data were first averaged within subjects, so that all subjects would contribute with equal weight to the estimates of the circadian and sleep dependent influences on the various variables. Next the data were averaged over subjects. Statistical significance was assessed with repeated measures ANOVAs. Statistical significance was based on Huynh-Feldt adjusted degrees of freedom, but the original degrees of freedom are reported. When missing data were present in some of the cells, a general ANOVA was applied, since a repeated ANOVA would require omitting all the data for those subjects for whom missing data were present in one or more cells. For some variables (e.g., sleep latency) data were log transformed in order to meet the requirements of a normal distribution.

\section{Results}

Circadian variation in sleep parameters

Distribution of sleep episodes over the cycle of core body temperature. During the forced desynchrony protocol, the period of

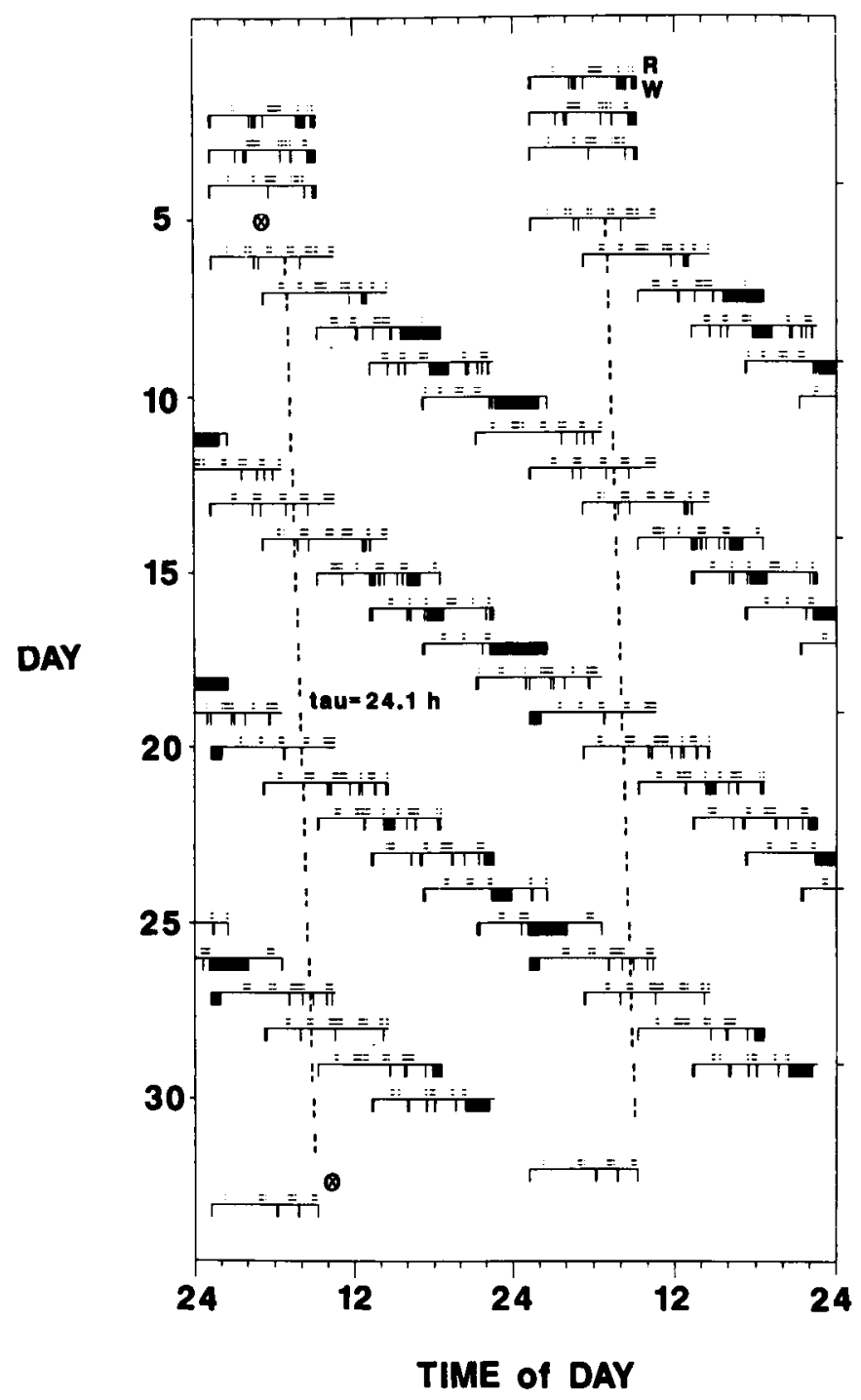

Figure 1. Double plot of the forced desynchrony protocol. Successive days are plotted both next to and beneath each other. In each sleep episode (horizontal solid line) wakefulness (solid black bars below sleep episode), and REM sleep (dotted lines/bars above sleep episode) are indicated for subject 1136 . An intrinsic circadian temperature cycle with a period of $24.1 \mathrm{hr}$ is estimated by a non-parametric spectral analysis of the core body temperature data during the forced desynchrony part of the protocol. The time of the minimum of the circadian temperature rhythm as estimated by the nonparametric spectral analysis, is indicated by the dashed line. The minimum of the endogenous circadian rhythm of core body temperature as assessed in the initial and final constant routine is indicated by an encircled $X$. The data are plotted with respect to clock time.

the core body temperature rhythm was different from both 24 and $28 \mathrm{hr}$ in all subjects. A typical example of the progression of the estimate of the phase of the minimum of the CBT rhythm during the experiment is given in Figure 1. In this subject the initial phase of the endogenous minimum of the core body temperature rhythm was located at 4:51 A.M. whereas $27 \mathrm{~d}$ later during the final constant routine it occurred at 10:38 hr. From this phase shift a period of $24.2 \mathrm{hr}$ can be derived. The estimate of period as assessed from the nonparametric spectral analysis of core body temperature data was $24.1 \mathrm{hr}$ which was close to the estimate derived from nonorthogonal spectral analysis (24.1 $\mathrm{hr}$ ). The phase of the minimum as estimated from nonparametric 

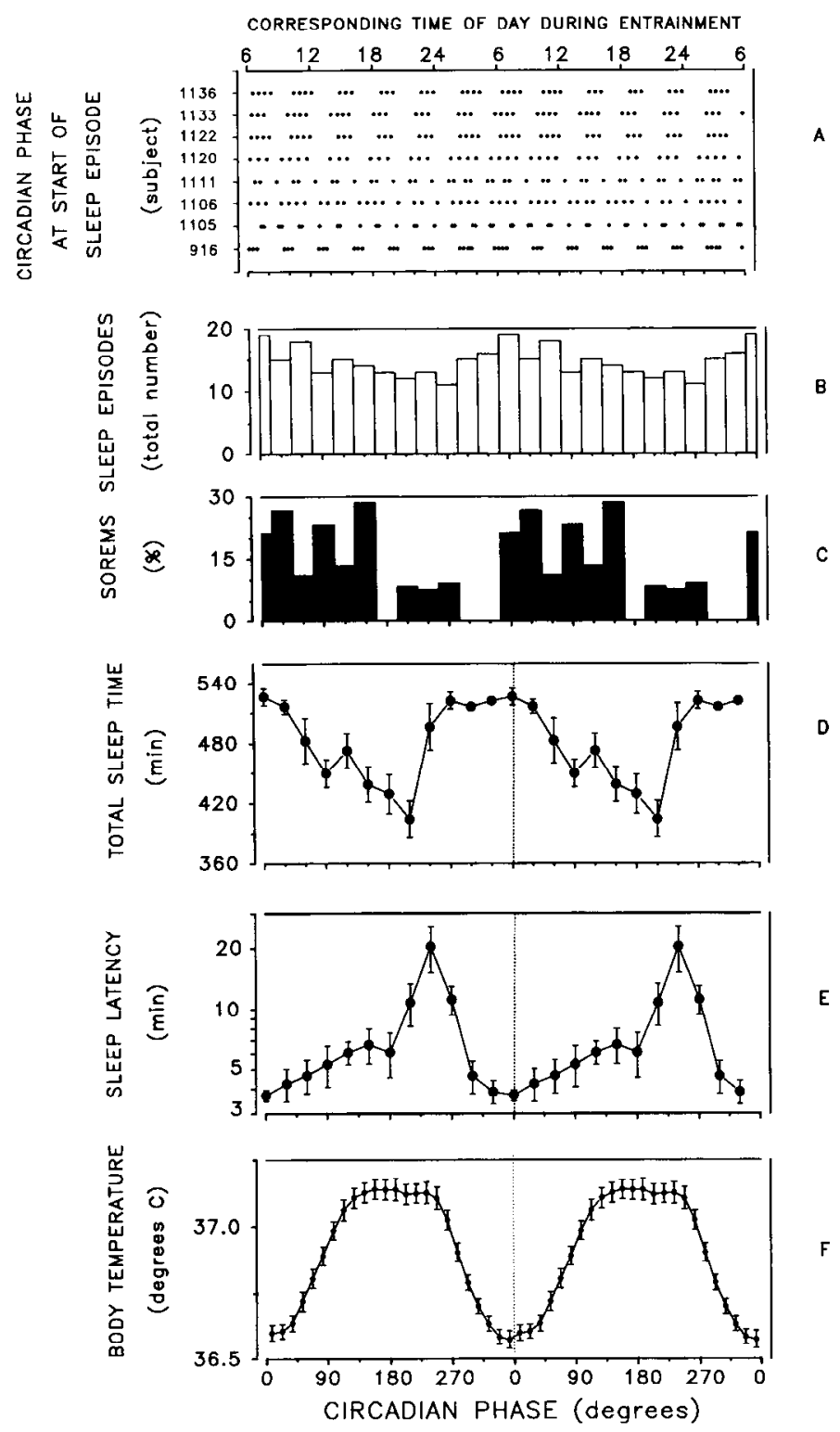

Figure 2. A, Distribution of the start of sleep episodes (i.e., lights out; $n=174$ ) over the circadian cycle for individual subjects. $B$, Circadian distribution of Number of Sleep Episodes begun. $C$, Circadian distribution of sleep-onset REM sleep episodes (interval between sleep latency and first occurrence of REM sleep $<10 \mathrm{~min}$ ). $D$. Total sleep time as a function of circadian phase at mid-sleep. Data were first for each bin averaged per subject and next averaged over subjects. $E$, Sleep latency as a function of circadian phase at lights out. Data were first $\log$ transformed then for each subject averaged per $30^{\circ}$ bin. Next the retransformed data were averaged per $30^{\circ}$ bin (i.e., 2 circadian hours) over subjects. $f$, Educed circadian wave form of core body temperature. The mid-trough of the core body temperature curve was assigned a circadian phase of $0^{\circ}$. Data are double plotted at the midpoints of the bins in all panels.

spectral analysis is indicated by the dashed line in Figure 1. In this subject scheduled sleep episodes occurred at various circadian phases (Figs. 1, 2A). Calculated over all subjects, the distribution of the beginning (lights out) of the sleep episodes over the circadian cycle of the core body temperature was quite uniform (Fig. $2 B$ ), although in individual subjects some clustering occurred (Fig. 2A).

Sleep initiation. The interval between the time of lights out and the first occurrence of any sleep stage (stage one included) was defined as sleep latency. Although sleep could be initiated rapidly at all circadian phases (mean $=6.2 ;$ minimum $=0.5$; maximum $=49.0 \mathrm{~min}$ ), sleep latencies varied significantly with circadian phase $\left(F_{11.82}=6.18 ; p<0.0001\right)$ (Fig. $2 E$ ). Minimum sleep latencies were observed at the minimum of the endogenous component of the temperature rhythm (Fig. 2E). Thereafter, latencies increased gradually. A transitory, statistically nonsignificant $\left(p>0.05, t\right.$ test) decrease was observed at $165^{\circ}-195^{\circ}$. This decrease was followed by a sudden increase in sleep latency values. In the interval of $195^{\circ}-285^{\circ}$, sleep latencies were significantly ( $p<0.05$ in all cases) longer than in the interval $285^{\circ}-105^{\circ}$. After this phase of long sleep latencies, values dropped sharply. The circadian variation of sleep latency was asymmetrical. The rising limb was approximately 16 circadian hours long and coincided with the rising and plateau part of the educed wave form of core body temperature, which in the entrained state would correspond to the interval from 6:00 to 22:00 hr. The falling limb was approximately 8 circadian hours long and coincided with the falling limb of the core body temperature rhythm, which in the entrained state would correspond to the interval from 22:00 to $06: 00 \mathrm{hr}$. The circadian variation of the latency to stage 2 (data not shown) was also significant $\left(F_{11,82}=5.54 ; p<0.0001\right)$ and very similar to sleep latency. The interval between lights out and the first occurrence of stages 3 or 4 (SWS) varied significant over the circadian cycle $\left(F_{11,82}\right.$ $=2.66 p<0.01$ ) but the circadian variation in the duration of the interval between sleep onset and the first occurrence of SWS was not significant $\left(F_{11.82}=1.16 \mathrm{NS}\right.$; data not shown).

Sleep onset REM sleep episodes (i.e., interval of sleep onset to the first occurrence of REM sleep $<10 \mathrm{~min}$ ) primarily occurred when sleep started at or shortly after the minimum of the core body temperature rhythm (Fig. $2 C$ ) although a $\chi^{2}$ statistics did not reach significance $\left(\chi^{2}=15.41 ; \mathrm{df}=11 ; p=0.16\right)$.

Sleep duration. Total sleep time (TST) obtained in the scheduled sleep episodes and sleep efficiency varied significantly with circadian phase $\left(F_{11,71}=8.02 ; p<0.0001\right.$; Fig. $2 D$; see also Fig. 1). TST and sleep efficiency were highest when the midpoint of the scheduled sleep episodes were located on the falling limb of the core body temperature curve with a maximum sleep efficiency of $94.0 \%(\mathrm{SEM}=1.5)$ at $0^{\circ}$. TST and sleep efficiency gradually decreased as the midpoint of the scheduled sleep episodes approached the maximum and plateau phase of the curve body temperature rhythm with a minimum sleep efficiency of $72.2 \%(\mathrm{SEM}=3.2)$ at $210^{\circ}$. Note that the falling limb of the TST rhythm was longer than the rising limb.

\section{Separation and interaction of circadian and sleep dependent components}

First, we estimated the strength and waveform of the circadian and sleep-dependent components by folding the data at either the endogenous circadian period or at the period of the restactivity cycle (Fig. 3). Next, we analyzed how these two processes interact in the regulation of the various variables. First the time course of these variables within sleep episodes starting at different circadian phases was analyzed. This analysis highlights the dependency of the sleep dependent component on circadian phase (Fig. 5). Next the circadian waveform of the different variables was investigated for various sections of the sleep episodes. This analysis emphasizes the dependency of the circadian modulation of sleep variables on sleep pressure (Fig. 6).

Wakefulness within scheduled sleep episodes. The circadian 


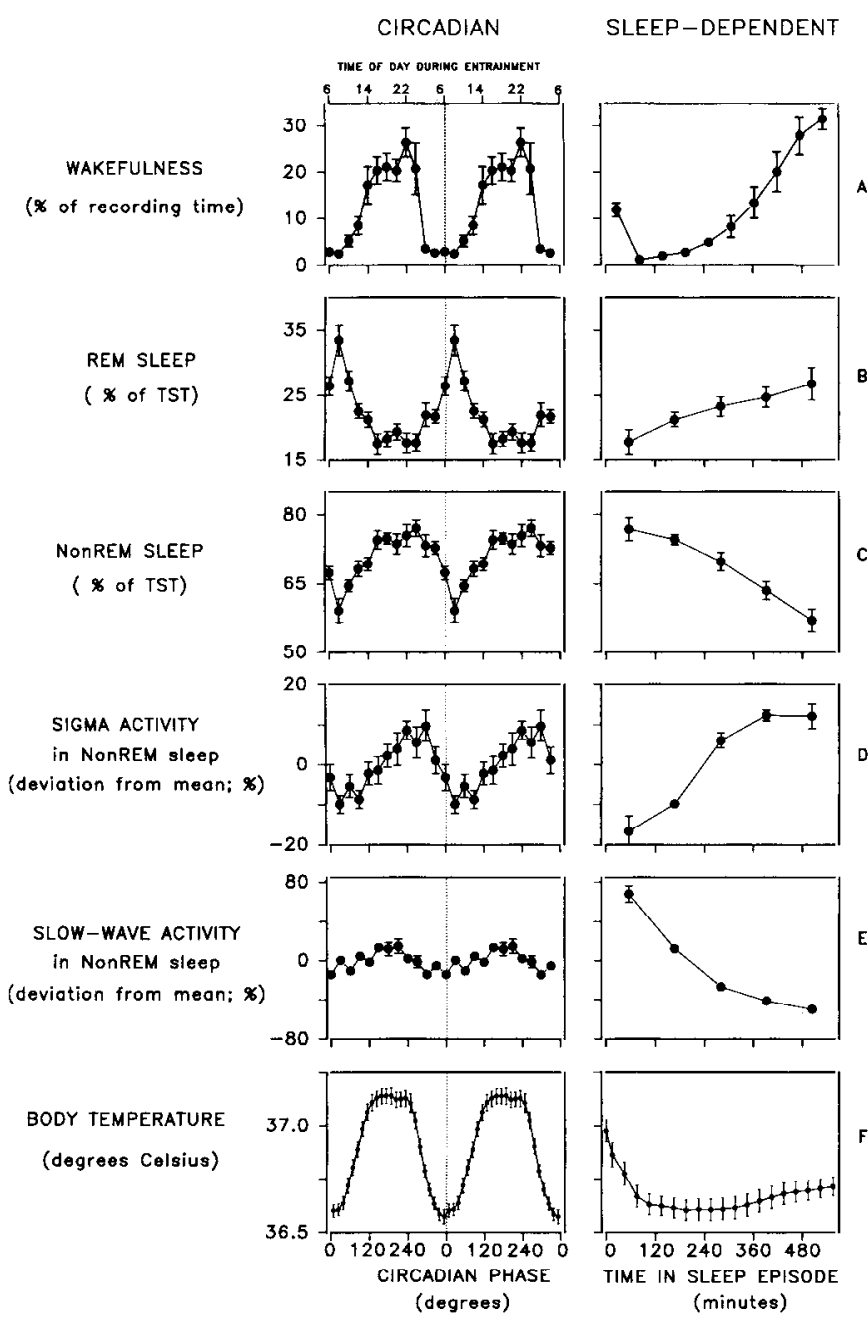

Figure 3. Separation of circadian (left) and sleep dependent components (right). Circadian wave-forms were educed by assigning data to circadian bins irrespective of time since start of sleep episode. Sleep dependent changes were educed by determining the time elapsed since the start of sleep episodes and assigning the data to $112 \mathrm{~min}$ bins, irrespective of circadian phase. Circadian waveform data are double plotted at the midpoints of the bins. $A$, Wakefulness in scheduled sleep episodes expressed as a percentage of recording time. $B$, REM sleep expressed as a percentage of total sleep time. $C$, Non-REM sleep expressed as a percentage of total sleep time. $D$, Sigma activity (i.e., sleep spindle activity: $12.75-15.0 \mathrm{~Hz}$ ) in non-REM sleep. Data were for each subject expressed as a deviation of its mean value and then averaged over subjects. $E$, Slow wave activity $(0.75-4.5 \mathrm{~Hz})$ in non-REM sleep. Data were for each subject expressed as a deviation of its mean value and then averaged over subjects. $F$, Core body temperature. The first point in the sleep dependent graph represents the average value during the last 30 min before lights out.

modulation of wakefulness within scheduled sleep episodes was significant $\left(F_{11,77}=16.94 ; p<0.0001 ;\right.$ rANOVA). Wakefulness was lowest around the nadir of the core body temperature rhythm (Fig. 3A, left). It increased on the rising limb and was maximal 15-17 circadian hours after the minimum of the core body temperature rhythm, whereafter wakefulness dropped sharply.

After an initial drop associated with the latency to sleep onset, wakefulness within the scheduled sleep episode increased as time since start of sleep episodes progressed $\left(F_{9,63}=28.10 ; p\right.$ $<0.0001$; rANOVA; Fig. $3 A$, right). The amplitude of the cir- cadian and sleep-dependent component were approximately similar.

In sleep episodes which were initiated at the minimum of the core body temperature rhythm, wakefulness was at low levels in the first two $112 \mathrm{~min}$ intervals but rose rapidly thereafter (Fig. 5). This rise became steeper when sleep was initiated on the rising limb of the endogenous core body temperature rhythm. When sleep was initiated at $180^{\circ}$, wakefulness was already at high levels in the $3 \mathrm{rd} 112 \mathrm{~min}$ interval (at approximately $240^{\circ}$ ), but decreased in the fourth interval, which was located on the falling limb of the core body temperature rhythm. When sleep was initiated at $210^{\circ}$, wakefulness was at approximately $10 \%$ in the first interval remained low throughout the sleep episode. In the sleep episodes initiated between $255^{\circ}$ and $285^{\circ}$, which would correspond to the clock-time interval from 23:00 to $01: 00 \mathrm{hr}$, wakefulness was below $10 \%$ in all intervals throughout the entire sleep episode.

The wave-form of the circadian modulation of wakefulness within scheduled sleep changed as a function of prior sleep (Fig. 6). During the first 112 min interval very little wakefulness was observed, independent of circadian phase except at $240^{\circ}$ where wakefulness was approximately $20 \%$. As sleep progressed wakefulness gradually increased and the circadian modulation became more pronounced. In the interval $336-448$ min after the start of sleep episodes, wakefulness gradually increased on the rising limb of the body temperature rhythm until a maximum was reached at $240^{\circ}$, that is, $15-17$ circadian hours after the minimum of the body temperature rhythm. After this phase wakefulness within the sleep episode dropped sharply. In the last interval (448-560 minutes) the circadian waveform was different: wakefulness started to increase $4 \mathrm{hr}$ after the minimum of CBT and reached high values on the rising and plateau phase of the core body temperature rhythm. When slecp coincided with the endogenous minimum of the core body temperature rhythm wakefulness remained at very low levels. A repeated measures ANOVA with factors "time elapsed since start of sleep episode" and "circadian phase" yielded a significant effect for both factors (time: $F_{4,28}=23.33 ; p<0.0001$; phase: $F_{11.77}=15.16 ; p$ $<0.0001)$ and a significant interaction between these two factors $\left(F_{44.308}=4.92 p<0.0001\right)$.

In all subjects the circadian drive for wakefulness was low around the minimum of the core body temperature rhythm and in none of the subjects was there a pronounced increase of wakefulness before $4-6 \mathrm{hr}$ after the minimum of the core body temperature rhythm (Table 1).

Individual differences in both amplitude and waveform were present. Whereas in four subjects the strongest drive for wakefulness was located at approximately $240^{\circ}$, in others the crest was located at $180^{\circ}-200^{\circ}$. Evidence for a bimodal waveform of the circadian drive for wakefulness rhythm was present in some subjects although in none of the subjects could a strong drive for sleep be detected around the maximum of the core body temperature rhythm.

REM sleep. A pronounced circadian modulation of REMS was observed $\left(F_{11.77}=17.33 ; p<0.0001\right.$; rANOVA; Fig. $3 B$, left) with maximum values of approximately $35 \%$ of TST. This maximum was located shortly after the minimum of the endogenous rhythm of core body temperature. Hereafter, REMS dropped sharply with minimum values of approximately $17 \%$ of TST at the plateau phase of the core body temperature rhythm. The sleep dependent change in REMS when expressed as a percentage of TST, was significant $\left(F_{4,2 \mathrm{X}}=4.85 ; p<0.01\right.$; r- 
Slgma Activity

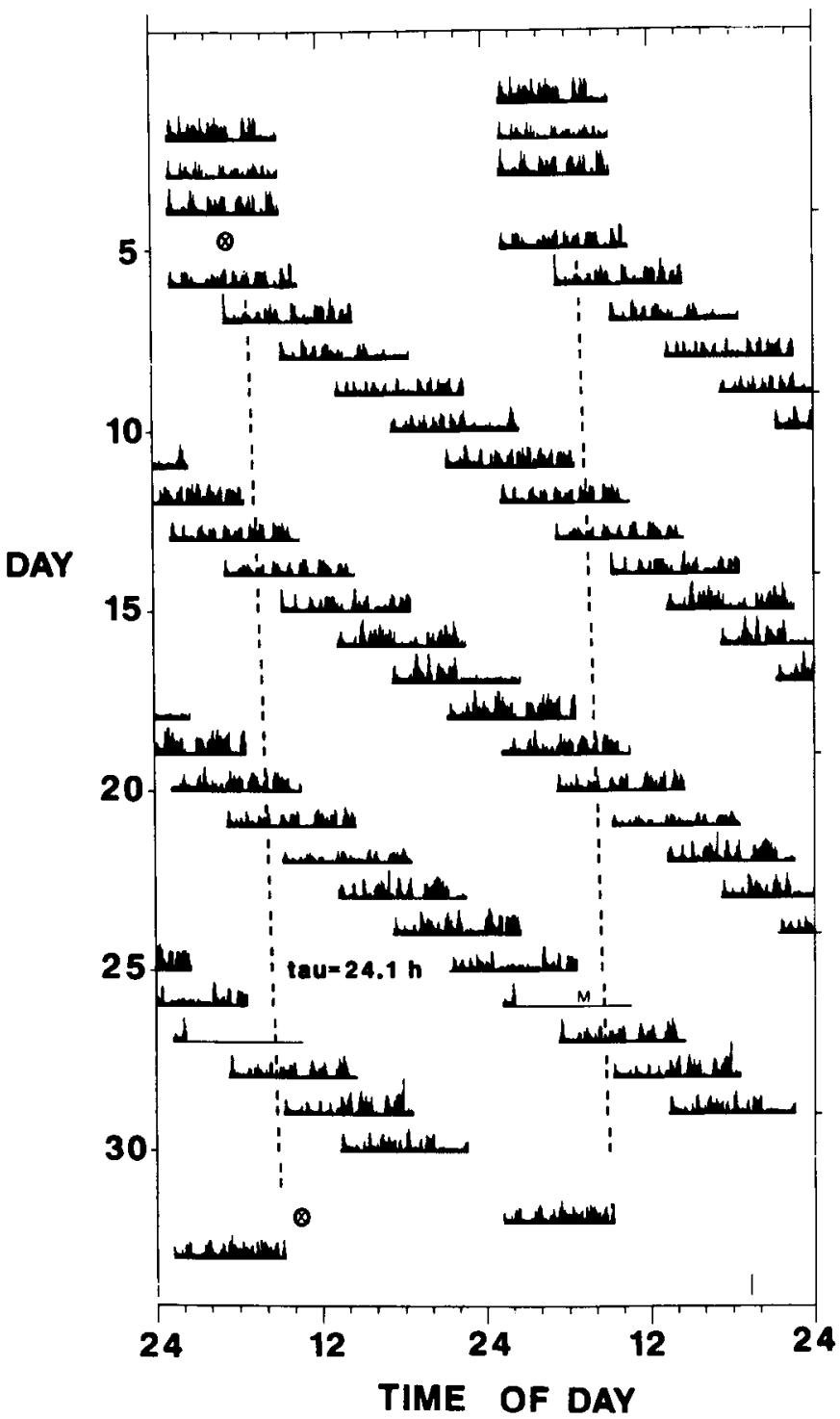

B Slow Wave Activity

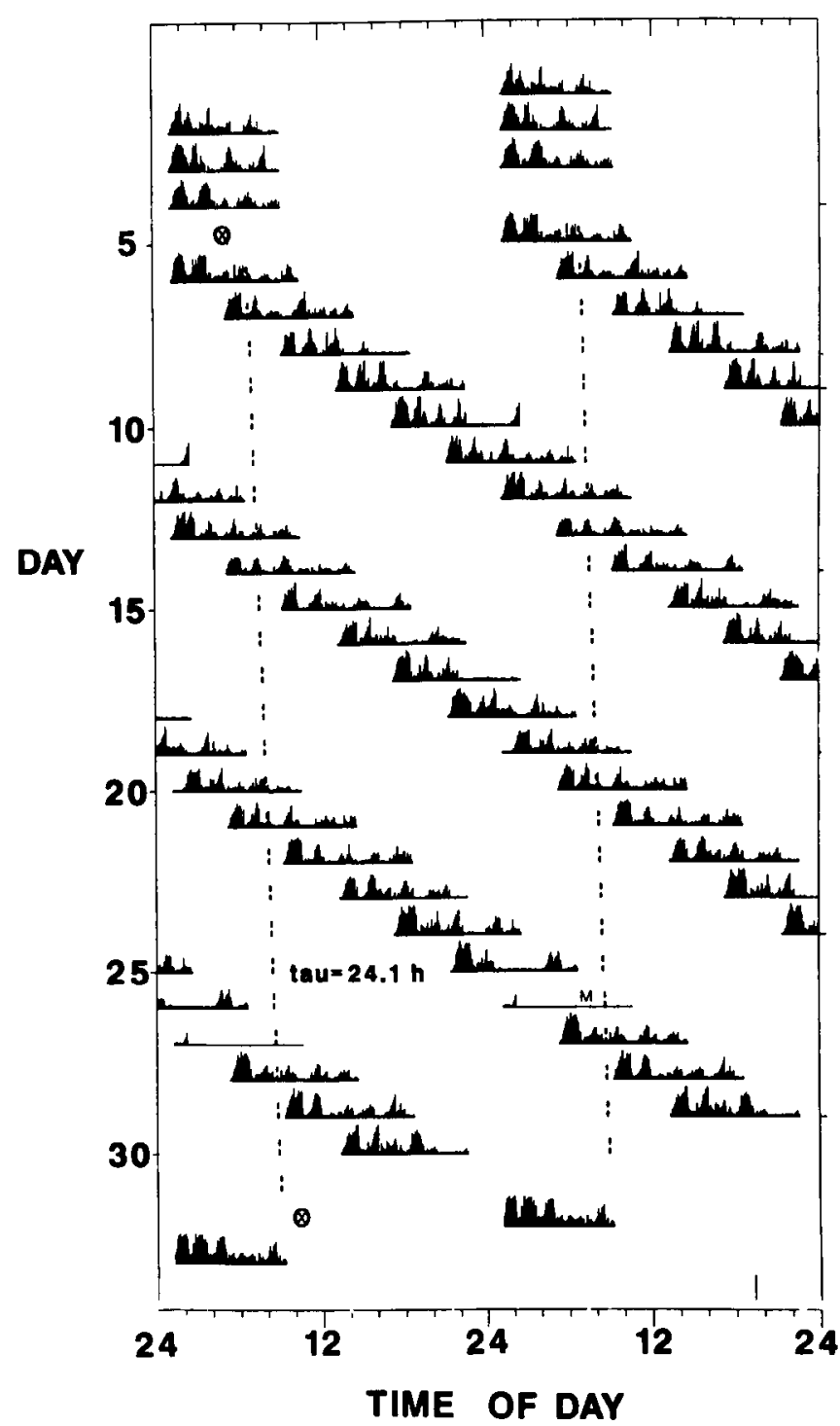

Figure 4. Double plot of the time course of sigma activity and slow-wave activity during sleep episodes in the forced desynchrony protocol (subject 1136). Artifacts during sleep and wakefulness within the scheduled sleep episodes were removed. One minute averaged values are plotted for slow-wave activity. For sigma activity data were smoothed with a 3 min moving average procedure. The time of the minimum of the circadian temperature rhythm, estimated by nonparametric spectral analysis, is indicated by the dashed line. Successive days are plotted both next to and beneath each other. Each minimum of the endogenous circadian rhythm of core body temperature as assessed in the initial and final constant routine is indicated by an encircled X. $M$ indicates missing data. The data were plotted with respect to clock time. The vertical lines in the right-hand lower corner correspond to $2 \mu \mathrm{V}^{2} / \mathrm{Hz}$ for sigma activity and $200 \mu \mathrm{V}^{2} / \mathrm{Hz}$ for slow-wave activity.

ANOVA). REMS gradually increased over the five consecutive 112 min intervals of sleep (Fig. $3 B$, right). The time course of REM sleep within scheduled sleep episodes was strongly dependent on circadian phase (Fig. 5B). REM sleep increased in the course of sleep episodes, especially in sleep episodes initiated between $157.5^{\circ}$ and $337.5^{\circ}$. In sleep episodes initiated at and after the minimum of the core body temperature rhythm REM sleep decreased in the course of sleep. In sleep episodes starting between $157.5^{\circ}$ and $292.5^{\circ}$ REM sleep values in the initial part of sleep episodes were lower compared to values in the later part of sleep episodes at approximately the same circadian phase (see also Fig. 6).

A repeated measures ANOVA on the data presented in Figure $6 B$ confirmed that REM sleep was both determined by circadian phase $\left(F_{11,77}-24.66 ; p<0.0001\right)$ and time elapsed since sleep onset $\left(F_{4,28}=3.74 ; p<0.02\right)$. Furthermore, a significant interaction between these two factors was present $\left(F_{44,308}=1.85 ; p<0.01\right)$.

Non-REM sleep. Non-REMS (Stage $2+3+4$ ) expressed as a percentage of TST exhibited a significant circadian modulation $\left(F_{11,77}=14.68 ; p<0.0001 ;\right.$ rANOVA $)$. Highest values were observed at approximately $240^{\circ}$, that is, shortly after the plateau phase of the endogenous core body temperature rhythm, with a maximum value close to $80 \%$ of TST. Non-REMS decreased on the falling limb of the core body temperature rhythm and lowest values were located immediately after the minimum of the core body temperature rhythm (Fig. $3 C$ ). The sleep dependent decrease of non-REMS was significant $\left(F_{4.28}=26.07 ; p<0.0001 ; \mathrm{r}\right.$ ANOVA; Fig. $3 C$, right) 
WAKEFULNESS

(\% of RT)

REM SLEEP

(\% of RT)

SIGMA ACTIVITY

in NonREM sleep

(deviation from mean; $\%$ )

\section{SLOW-WAVE ACTIVITY}

in NonREM sleep

(deviation from mean; $\boldsymbol{x}$ )

\section{BODY TEMPERATURE}

(degrees Celsius)
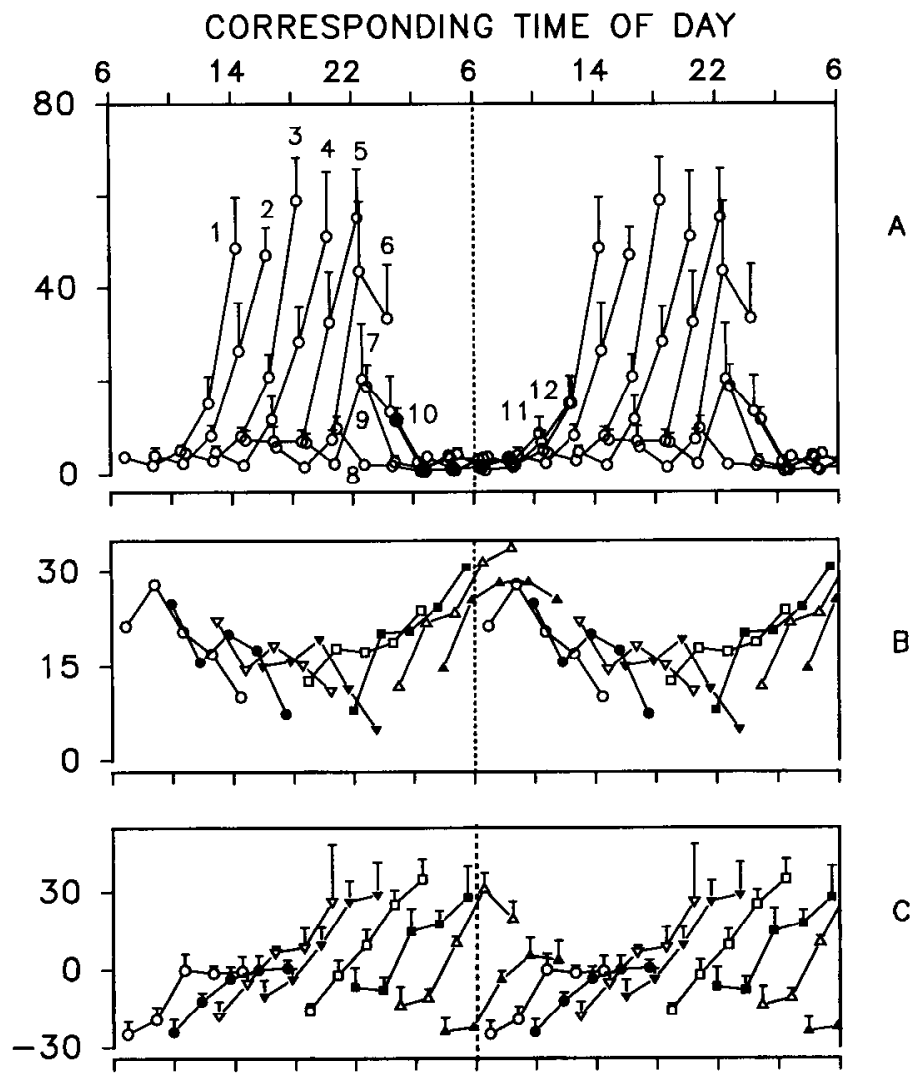

C

D

E

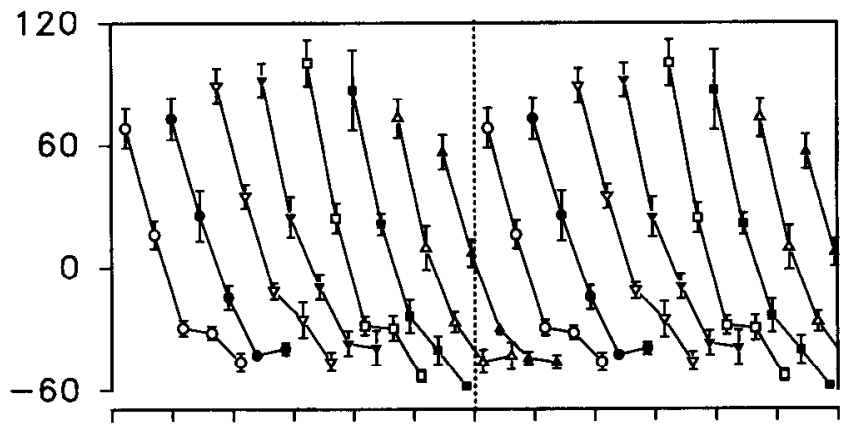

36.25

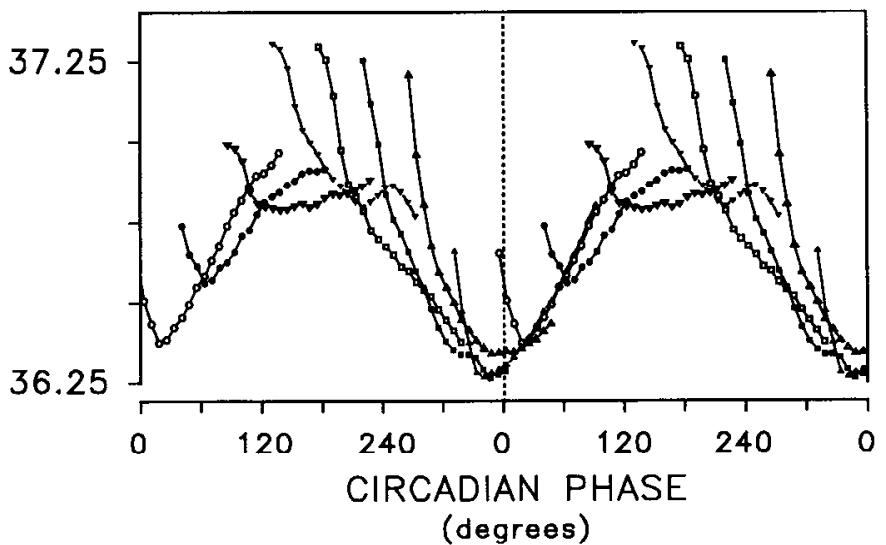

Figure 5. Sleep-dependent changes as a function of circadian phase. In $A$ sleep episodes were assigned to $1230^{\circ}$ bins based on the circadian phase at lights out. In $B E$ sleep episode were assigned to eight $45^{\circ}$ bins. Legend for symbols in $B E: \bigcirc,-22.5^{\circ}$ to $22.5^{\circ} ; 0,22.5^{\circ}-67.5^{\circ} ; \nabla$, $76.5^{\circ}-112.5^{\circ} ; \nabla, 112.5^{\circ}-157.5^{\circ} ; \square, 157.5^{\circ}-202.5^{\circ} ; \square, 202.5^{\circ}-247.5^{\circ} ; \triangle, 247.5^{\circ}-292.5^{\circ} ; \boldsymbol{\Delta}, 292.5^{\circ}-337.5^{\circ}$. A, Wakefulness in schedules sleep episodes, expressed as a percentage of recording time. O. Sleep episodes initiated between $255^{\circ}$ and $285^{\circ}$ (23-01 hr), which corresponds to the habitual tining of sleep unset during entrained conditions. Numbers in graph identify bin number: $1,-15^{\circ}-15^{\circ} ; 2,15^{\circ}-45^{\circ} ; 3,45^{\circ}-75^{\circ} ; 4,75^{\circ}-$ $105^{\circ} ; 5,105^{\circ}-135^{\circ} ; 6,135^{\circ}-165^{\circ} ; 7,165^{\circ}-195^{\circ} ; 8,195^{\circ}-225^{\circ} ; 9,225^{\circ}-255^{\circ} ; 10,255^{\circ}-285^{\circ} ; 11,285^{\circ}-315^{\circ} ; 12,315^{\circ}-345^{\circ}$. B, REM sleep, expressed as a percentage of recording time. $C$, Sigma activity in non-REM sleep, expressed as a deviation from each individual's mean value. $D$, Slow wave activity in non-REM sleep, expressed as a deviation from each individual's mean value. $E$, Core body temperature. Note that the first value of each temperature curve represents the average core body temperature during the last $30 \mathrm{~min}$ before lights out. 
WAKEFULNESS

( $X$ of RT)
REM SLEEP

(\% of RT)
SIGMA ACTIVITY

(deviation from mean; $x$ )
SLOW WAVE ACTIVITY

(doviation from mean;:)
BODY TEMPERATURE

(degrees Colsius)
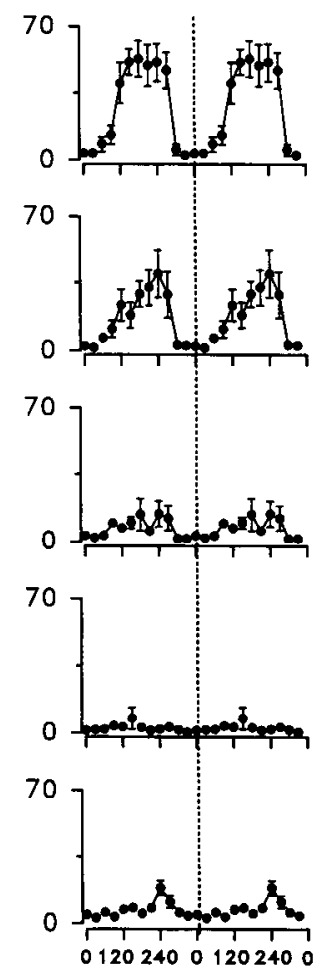
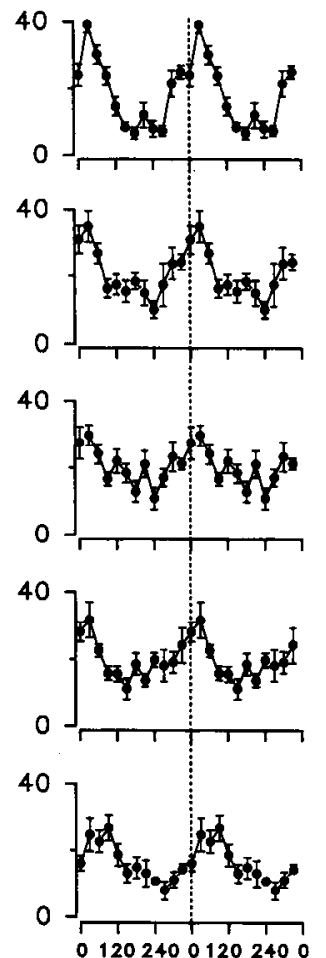
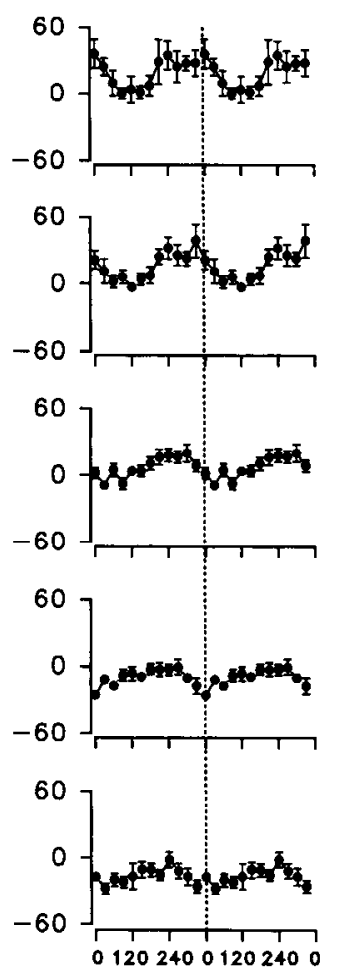
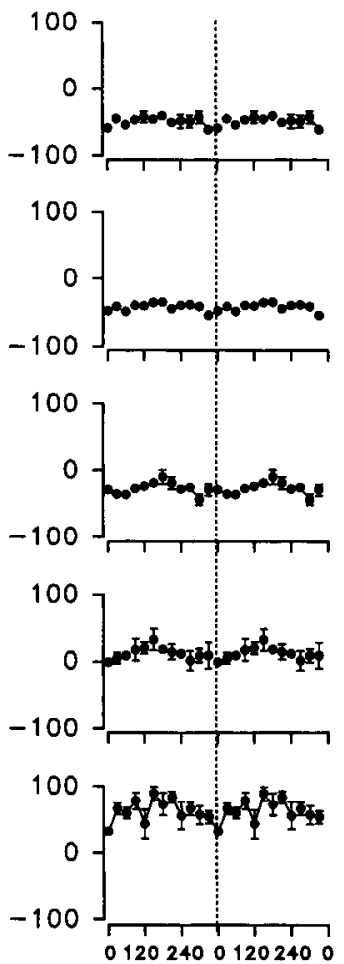
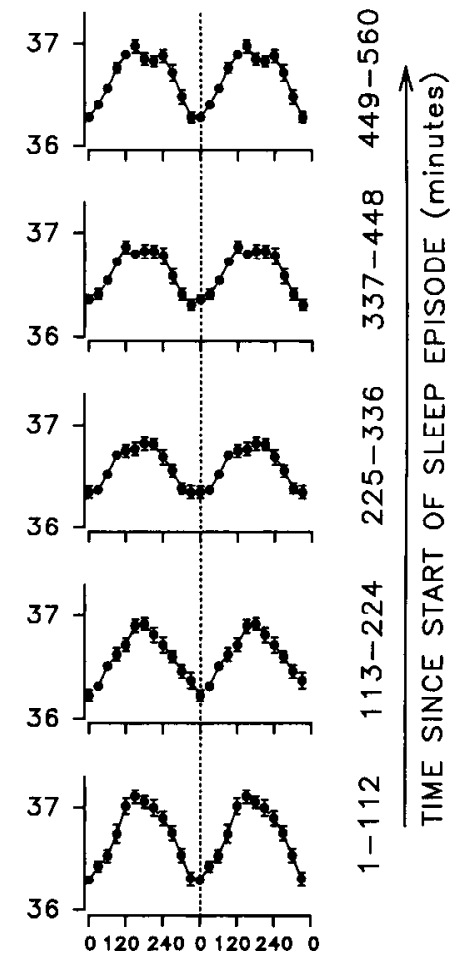

CIRCADIAN PHASE (degrees)

Figure 6. Circadian modulation of sleep parameters as a function of time elapsed since start of sleep episode.The circadian modulation was calculated separately for five 112 min segments of sleep episodes. $A$, Wakefulness in sleep episodes, expressed as a percentage of recording time. $B$, REM sleep, expressed as a percentage of recording time. C, Sigma activity in non-REM sleep, expressed as a deviation from each individual's mean value. $D$, Slow wave activity in non-REM sleep, expressed as a deviation from each individual's mean value. $E$, Core body temperature.

Sigma activity (sleep spindle activity) in non-REM sleep. The time course of sigma activity $(12.75-15.0 \mathrm{~Hz})$ is illustrated in Figure $4 A$ for subject 1136 . In baseline sleep (Fig. 4, sleep episode 3) sigma activity exhibits its typical time course: in the

\begin{tabular}{|c|c|c|c|c|c|c|c|c|}
\hline \multirow{2}{*}{$\begin{array}{l}\text { Phase } \\
\text { (time) }\end{array}$} & \multicolumn{8}{|c|}{ Subject } \\
\hline & 916 & 1105 & 1106 & 1111 & 1120 & 1122 & 1133 & 1136 \\
\hline $0(06: 00 \mathrm{hr})$ & 3.9 & 7.1 & 1.4 & 2.6 & 2.6 & 2.3 & 2.5 & 0.5 \\
\hline $30(08: 00 \mathrm{hr})$ & 2.7 & 6.3 & 0.7 & 1.4 & 3.9 & 1.6 & 1.2 & 1.6 \\
\hline $60(10: 00 \mathrm{hr})$ & 12.4 & 6.5 & 3.0 & 4.3 & 7.5 & 4.7 & 2.5 & 1.2 \\
\hline $90(12: 00 \mathrm{hr})$ & 13.2 & 7.8 & 4.3 & 6.5 & 19.9 & 4.7 & 3.3 & 8.4 \\
\hline $120(14: 00 \mathrm{hr})$ & 26.7 & 35.5 & 8.6 & 5.0 & 28.3 & 11.3 & 13.6 & 8.8 \\
\hline $150(16: 00 \mathrm{hr})$ & 14.1 & 26.7 & 13.4 & 15.7 & 33.1 & 25.3 & 8.9 & 25.4 \\
\hline $180(18: 00 \mathrm{hr})$ & 26.9 & 28.2 & 8.4 & 21.1 & 32.0 & 18.3 & 10.9 & 23.3 \\
\hline $210(20: 00 \mathrm{hr})$ & 16.2 & 27.1 & 11.9 & 21.6 & 30.0 & 12.2 & 24.7 & 19.5 \\
\hline $240(22: 00 \mathrm{hr})$ & 21.6 & 42.3 & 24.3 & 15.6 & 23.7 & 20.2 & 27.1 & 36.5 \\
\hline $270(24: 00 \mathrm{hr})$ & 17.4 & 43.9 & 9.0 & 4.8 & 20.0 & 17.1 & 9.1 & 45.1 \\
\hline $300(02: 00 \mathrm{hr})$ & 5.4 & 5.8 & 0.7 & 2.2 & 2.3 & 0.6 & 3.8 & 6.9 \\
\hline $330(04: 00 \mathrm{hr})$ & 2.7 & 4.0 & 1.2 & 4.3 & 3.5 & 1.1 & 2.3 & 1.9 \\
\hline
\end{tabular}

Data are calculated per $30^{\circ}$ bins. Values at $0^{\circ}$ represent wakefulness in scheduled sleep coinciding with circadian phase $-15^{\circ}$ to $15^{\circ}$, which corresponds to the interval of approximately 5:00 to 7:00 hr during entrainment. first non-REM sleep episodes high values can be observed at the beginning and end of the non-REM sleep episodes, whereas in later non-REM sleep episodes sigma activity remains high throughout the episodes. When averaged over all subjects and sleep episodes $(n=147)$, the sleep dependent increase was statistically significant $\left(F_{4,24}=22.61 ; p<0.001 ;\right.$ rANOVA) (Fig. $3 D$, right). Sigma activity exhibited a pronounced and significant $\left(F_{11,66}=3.65 ; p<0.01\right.$; rANOVA $)$ circadian rhythm with its crest at around 240-300 degrees, that is, coincident with the initial part of the habitual sleep episode (Fig. $3 D$, left). Sigma activity increased in all sleep episodes (Fig. $5 C$ ) and the largest increase was observed when sleep was initiated on the rising limb of the core body temperature rhythm. The interaction between the circadian and sleep dependent component was complex $\left(F_{44.353}=1.32 ; p<0.1\right)$ as is illustrated in Figure $6 C$. In the initial part of sleep the circadian maximum of sigma activity was located at approximately $240^{\circ}$. As sleep progresses high values were located also around the minimum of the core body temperature rhythm.

Slow-wave activity in non-REM sleep. The time course of slow-wave activity (SWA; power density in the frequency range of $0.75-4.5 \mathrm{~Hz}$ ) in all sleep episodes was plotted with a one minute time resolution for subject 1136 (Fig. 4B). SWA exhibited ultradian oscillations, with high values during non-REM sleep and low values during REM sleep and wakefulness. Despite the observed variations in non-REM sleep and REM sleep 


\begin{tabular}{|c|c|c|}
\hline Phase & SWA & TST \\
\hline 0 & $168.2(9.5)$ & $524.7(4.2)$ \\
\hline 45 & $172.9(10.3)$ & $492.9(17.5)$ \\
\hline 90 & $189.1(8.7)$ & $458.3(13.9)$ \\
\hline 135 & $192.0(8.3)$ & $443.5(17.3)$ \\
\hline 180 & $200.3(11.3)$ & $397.4(21.6)$ \\
\hline 225 & $187.0(19.6)$ & $475.0(25.6)$ \\
\hline 270 & $173.0(9.4)$ & $527.8(5.9)$ \\
\hline 315 & $156.5(8.4)$ & $518.7(3.3)$ \\
\hline
\end{tabular}

SWA is normalized to the individual average value calculated over all sleep episodes and expressed as percentage. Total sleep time is expressed in minutes. Values in parentheses represent SEMs. SWA data were assigned to bins according to circadian phase of start of sleep episode. Data in the row labeled Phase 0 represent SWA in non-REM sleep in the first 56 min of sleep of sleep episodes which started between $-22.5^{\circ}$ and $22.5^{\circ} . n=7$ for all cells.

episode duration (see Fig. 1), SWA showed a global declining trend in the course of all sleep episodes. Some variation in the initial values of SWA could be observed. Averaged over all sleep episodes SWA in non-REM sleep exhibited a small but statistically significant variation with circadian phase $\left(F_{11.66}=\right.$ $6.16 ; p<0.0001$; rANOVA, Fig. $3 E$ ). Lowest values were observed around the minimum of the core body temperature rhythm and highest values were present at $150^{\circ}$. Note that since SWA is a density measure the observed variation cannot directly be relatcd to variation in the duration of non-REM slecp which exhibited circadian variation (Fig. $3 C$ ). SWA in non-REM sleep, exhibited a pronounced decline over consecutive 112 min intervals $\left(F_{4.24}=91.86 ; p<0.0001\right.$; rANOVA; Fig. $\left.3 E\right)$. Fitting the data to an exponential function $\left(\mathrm{SWA}_{t}=\mathrm{SWA}_{0} * e^{(-t / \tau)}+\mathrm{SWA} \infty\right.$; with $t=$ time in minutes) yielded a time constant $(\tau)$ of 185.9 (95\% confidence interval: 123.2-248.8).

SWA decreased in all sleep episodes independent of circadian phase, but the initial values were somewhat higher when sleep was initiated on the rising limb of the core body temperature rhythm as compared to sleep episodes initiated on the falling limb of this rhythm (Fig. 5D; see also Fig. 6D). An ANOVA with factors "time elapsed since sleep onset" and "circadian phase" revealed a significant effect of the factor "time" ( $F_{4,353}$ $=301.65 ; p<0.0001)$ and a significant effect for the factor "circadian phase" $\left(F_{11.353}=3.08 p<0.001\right)$, but the interaction between the two factors was not significant $\left(F_{44,353}=0.73\right.$; NS). For all sleep episodes in which SWA was assessed the duration of sleep (TST) in the preceding episode was calculated. The curve for TST in preceding sleep episodes mirrored the observed circadian modulation of SWA in the initial interval of sleep episodes (Table 2). Calculated over all sleep episodes, a small but statistically significant negative correlation between TST in the preceding sleep episode and SWA (rank ordered within each subject) in the initial 112 min of the subsequent sleep episode was observed (Pcarson's corrclation coefficient $=-0.27715 ; p$ $<0.001 ; n=140$ ).

Core-body temperature. The circadian waveform of core body temperature was characterized by a narrow trough and a broader maximum (Fig. $3 F$ ). The sleep dependent change of core body temperature $\left(F_{19,114}=22.60 ; p<0.0001\right.$; rANOVA) was characterized by a initial rapid drop and a subsequent slow and sta- tistically significant ( $p<0.01$; Helmert contrast) increase starting approximately $4 \mathrm{hr}$ after the beginning of the sleep episode.

The time course of body temperature during scheduled sleep episodes was strongly dependent on circadian phase (Fig. 5E). When sleep was initiated at or shortly after the endogenous the minimum of the CBT rhythm, CBT exhibited an initial small decline which was followed by a subsequent rise. As a result of the initial drop associated with the start of the sleep episode, the observed minimum of CBT was located up to 60 degrees after the endogenous minimum. When sleep was initiated at approximately $240^{\circ}$, CBT decreased steeply throughout the entire sleep episode. Note that when sleep was initiated at approximately $180^{\circ}$ the drop of temperature from lights out to the first $30 \mathrm{~min}$ interval was very small.

ANOVA of the data presented in Figure $6 E$ demonstrated a significant effect of both circadian phase $\left(F_{11.418}=76.28 ; p<\right.$ $0.0001)$ and time since start of slecp cpisode $\left(F_{4.418}=9.85 ; p\right.$ $<0.0001)$. Most noticeable is the smaller amplitude of the observed circadian rhythm of temperature in the middle of the sleep episode. The circadian waveform of core body temperature during sleep also appeared to change as a function of time since start of sleep episode, but the interaction between the factors circadian phase and time since start of sleep episode did not reach statistical significance $\left(F_{44,418}=1.16\right.$; NS).

\section{Discussion}

The present data confirm the pivotal role of the endogenous circadian pacemaker in the regulation of sleep in humans and show that sleep propensity and sleep structure result from interactions, which in part are nonadditive, of circadian and sleepwake dependent oscillatory processes. The data further demonstrate that sleep spindle activity in non-REM sleep is affected by endogenous circadian phase and that the endogenous circadian rhythm in the propensity to initiate sleep does not vary in parallel with slow-wave activity in the initial part of sleep.

\section{Sleep initiation, consolidation, and sleep duration}

The data underscore the close relation between the endogenous circadian rhythm of core body temperature and sleep regulation (Glotzbach and Heller, 1994). Our estimate of the circadian rhythm of sleep propensity, with its crest located at the minimum of the core body temperature rhythm (that is, close to habitual wake time) and its trough located close to habitual bed time, confirms results obtained in recent experiments in which the confound of prior wakefulness was minimized (Klein et al., 1993; Dantz et al., 1994) and previous estimates (Weitzman et al., 1974; Carskadon and Dement, 1975; Webb and Agnew, 1975; Czeisler, 1978; Lavie, 1986; Zulley, 1990). The analysis of wakefulness within scheduled sleep episodes unequivocally demonstrates that the probability to wake up from sleep results from an interaction between a circadian and a sleep dependent process, which is not simply additive since the shape and amplitude of the circadian wave-form of sleep efficiency depends on prior sleep duration. The data suggest that the signal which promotes wakefulness, gradually becomes stronger on the rising limb of the endogenous rhythm of corc body temperature, with a maximum at approximately $240^{\circ}$, which corresponds to the evening wake-maintenance zone (Strogatz et al., 1987) and the so-called "forbidden zone" for sleep (Lavie et al., 1986).

Since the circadian drive for wakefulness does not rise steeply until 4-8 hr after the minimum of the core-body temperature rhythm, spontaneous awakening at the habitual wake-time under 
entrained conditions must occur primarily because of prior slecp (Dijk and Czeisler, 1994) and is unlikely to be related to a strong circadian wake-up signal or morning wake maintenance zone (Strogatz et al., 1987).

\section{REM sleep, EEG slow-wave, and sleep spindle activity}

REM sleep was strongly modulated by circadian phase, with a remarkably narrow peak in circadian REM sleep propensity located shortly after the minimum of the body temperature rhythm (cf. Hume and Mills, 1977; Carskadon and Dement, 1980; Czeisler et al., 1980b; Zulley and Wever, 1982). This circadian modulation of REM sleep was of approximately equal strength throughout sleep, which contrasts McCarley's (1990) interpretation of previous data (Zulley, 1980). The sleep-dependent disinhibition of REM sleep interacting with the circadian rhythm of REM sleep results in very high amounts of REM sleep when the end of the sleep episode is located close to the minimum of the body temperature rhythm as occurs under entrained conditions. This may facilitate the rearousal of the sleeping brain, providing a gate from sleep to waking at our habitual wake-time (Snyder, 1966; Lavie et al., 1979; Wehr, 1992).

I'he sleep-dependent disinhibition of REM sleep and the sleep dependent decline in sleep propensity could be related to the decline of SWA in the course of sleep (Borbély et al., 1982), which, as the present data demonstrate, occurs independent of circadian phase (see also Weitzman et al., 1980; Åkerstedt and Gillberg, 1981; Gillberg and Åkerstedt, 1982). However, efforts to slow down the sleep dependent decline of sleep propensity by experimental manipulations of SWA have provided equivocal results (Dijk and Beersma, 1989; Gillberg et al., 1991).

The variation of SWA in the initial part of sleep, with higher values on the rising limb of the CBT rhythm (see also Campbell and Zulley, 1989; Zulley, 1990) may either represent a circadian modulation of SWA, possibly related to REM sleep or variations in the non-REM-REM cycle (Beersma et al., 1990; Brunner et al., 1990; Dijk et al., 1991). Alternatively, this variation in SWA may be associated with the circadian variation in sleep time in the preceding sleep episodes (Table 2). Although the data are not at variance with the hypothesis that slow-wave activity is an electrophysiological correlate of a sleep-wake dependent oscillatory process serving sleep homeostasis (Borbély, 1982; Daan et al., 1984, Achermann et al., 1993), they also demonstrate that the observed variation in SWA in the initial part of sleep does not vary in parallel with sleep propensity. Actually, only REM sleep peaked close to the crest of the sleep propensity rhythm (see also Broughton and Aguirre, 1987).

The sleep-dependent increase in sigma activity, which was present at all circadian phases, demonstrates that the previously reported inverse relationship between spindle activity and SWA during nocturnal sleep (Uchida et al., 1991; Aeschbach and Borbély, 1993; Dijk et al., 1993) is indeed primarily related to the progression of sleep and not to circadian phase. A new finding was the circadian modulation of sigma activity. Its average wave form was somewhat surprising in that the maximum of sigma activity (associated with the highest density of sleep spindles within non-REM sleep; Dijk et al., 1993) was located shortly after the peak of the wake propensity rhythm, coincident with habitual bed time. The analysis of the interaction between circadian and sleep dependent influence, however, showed that high values of spindle activity are present at the minimum of the core body temperature rhythm when it coincides with the end of scheduled sleep. A more detailed analysis of spindle ac- tivity is necded to resolve whether some of the present results are related to putative circadian and sleep dependent changes in the frequency of sleep spindles. Likewise, other EEG frequencies including the recently described slow oscillations with a frequency lower than $1 \mathrm{~Hz}$ (Steriade et al., 1993b) remain to be investigated.

\section{Relation to the physiology of sleep and circadian rhythms}

In the present analyses, circadian phase has been derived from core body temperature data. Recent evidence indicates that SCN lesions histologically verified as complete, result in a loss of the CBT rhythm; this indicates contrary to previous conclusions (Fuller et al., 1981, Satinoff and Prosser, 1988), that the SCN does indeed drive the body temperature rhythm (Edgar et al., 1993). In the present protocol, the period of the rhythms of plasma melatonin (Shanahan and Czeisler, 1991a) and cortisol (Czeisler et al., unpublished observations), which are known to be driven by the SCN (Moore and Eichler, 1974; Shanahan and Czeisler, 1991b; Buijs et al., 1993), is identical to the period of the body temperature rhythm. It therefore seems reasonable to assume that the circadian variation of sleep propensity and sleep structure are related to variations in the output of the SCN or hormonal rhythms, for example, melatonin, which are driven by the SCN.

The circadian modulation of REM sleep propensity is likely to be mediated by the indirect projections from the SCN to mesopontine nuclei such as the pedunculopontine tegmental nucleus (PPT) and the laterodorsal tegmental nucleus (LDT) (see McCarley and Massaquoi, 1992) which are involved in the generation of REM sleep. ACh originating in the aforementioned mesopontine nuclei and the nucleus basalis of the forebrain has been implicated in neocortical activation during REM sleep and wakefulness (Buzsaki et al., 1988; Steriade et al., 1990, 1991; McCormick, 1992; Metherate et al., 1992; McCarley and Massaquoi, 1992; Rainnie et al., 1994). Direct projections from the $\mathrm{SCN}$ to the nucleus basalis have not been described, although it has been suggested that indirect connections to this nucleus could be made via the paraventricular nucleus of the hypothalamus, a major target of SCN efferents (Watts, 1991). Interestingly, the SCN receive cholinergic input from the nucleus basalis and from the PPT and LDT (Bina et al., 1993), which together with the serotonergic projection from the raphe dorsalis may constitute potential pathways for a feedback of arousal state onto the circadian pacemaker.

Although important differences in the neurophysiological mechanism underlying slow wave and sleep spindle genesis exist (Steriade et al., 1991; for review, see Steriade et al., 1993a), the appearance of both types of activity in the macroscopic EEG are dependent on hyperpolarization of thalamocortical neurons. Steriade has hypothesized that as sleep deepens, the transition from spindle-dominated EEG patterns to slow wave-dominated EEG is accompanied by a progressive hyperpolarization of thalamocortical neurons. Conversely, the reduction in SWA and the increase in spindle frequency activity during the course of sleep is likely to be associated with reduced tonic hyperpolarization of thalamocortical neurons.

The decrease in SWA and the increase of sleep spindle activity in the course of sleep which was observed at all circadian phases indicates that the predicted and observed inverse time course is related to the progression of sleep rather than endogenous circadian phase. This time course may constitute an essential component of the sleep process (Steriade et al., 1993a). 
The differential magnitude of circadian and sleep dependent contribution to slow waves and sleep spindles further supports the notion of the existence of multiple systems involved in components of EEG synchronization (Steriade et al., 1994) and may indicate that the SCN directly influences thalamic nuclei such as the nucleus reticularis, involved in spindle genesis.

During entrainment the phase relation between the endogenous circadian rhythm of sleep propensity and the sleep-wake cycle is such that wakefulness coincides with the falling limb of the circadian sleep propensity rhythm (Czeisler et al., 1992; Dijk and Czeisler, 1994). Previously, a nap study demonstrated that when wakefulness preceding sleep is varied from 2 to $20 \mathrm{hr}$, slow wave activity in non-REM sleep increases progressively (Dijk et al., 1987a). Thus, under entrained conditions, a wakefulness-related increase in slow-wave propensity coincides with the circadian reduction in the propensity to initiate sleep. In accordance with model simulations (Borbćly ct al., 1989) and a hypothesis derived from observations in the SCN-lesioned squirrel monkey (Edgar et al., 1993) we suggest that in humans this paradoxical increase in the circadian drive for wakefulness during the course of the habitual waking day opposes the wakedependent increase in sleep propensity, deterioration of alertness (Dijk et al., 1992; Johnson et al., 1992) and slow-wave propensity. Thus, the suprachiasmatic nuclei seem to provide a signal which allows for a consolidated bout of wakefulness (Dijk and Czeisler, 1994) and EEG desynchronization, which is a sine qua non for optimal cognitive functioning, despite the increase in sleep propensity and EEG slow-wave propensity associated with wakefulness.

\section{References}

Achermann P, Dijk DJ, Brunner DP, Borbély AA (1993) A model of human sleep homeostasis based on EEG slow-wave activity: quantitative comparison of data and simulations. Brain Res Bull 31:97113.

Aeschbach D. Borbély AA (1993) All-night dynamics of the human sleep EEG. J Sleep Res 2:70-81.

Åkerstedt T, Gillberg M (1981) The circadian variation of experimentally displaced sleep. Sleep 4:159-169.

Åkerstedt T, Gillberg M (1986) Sleep duration and the power spectral density of the EEG. Electroencephalogr Clin Neurophysiol 64:119222.

Aschoff J (1965) Circadian rhythms in man. Science 148:1427-1432.

Aschoff J, Gerecke U, Wever R (1967) Desynchronization of human circadian rhythms. Jpn J Physiol 17:450-457.

Beersma DGM, Daan S, Dijk DJ (1987) Sleep intensity and timing: a model for their circadian control. In: Lectures on mathematics in the life sciences, Vol 19, Some mathematical questions in biology: circadian rhythms (Carpenter GA, ed), pp 39-62. Providence, RI: American Mathematical Society.

Beersma DGM, Dijk DJ, Blok G, Everhardus I (1990) REM sleep deprivation during five hours leads to an immediate REM sleep rebound and to suppression of non-REM sleep intensity. Electroencephalogr Clin Neurophysiol 76:114-122.

Bina KG, Rusak B, Semba K (1993) Localization of cholinergic neurons in the forcbrain and brainstem that project to the suprachiasmatic nucleus of the hypothalamus in rat. J Comp Neurol 335:295-307.

Borbély AA (1982) A two-process model of sleep regulation. Human Neurobiol 1:195-204.

Borbély AA, Baumann F, Brandeis D, Strauch I, Lehmann D (1981) Sleep deprivation: effect on sleep stages and EEG power density in man. Electroencephalogr Clin Neurophysiol 51:483-493.

Borbély AA, Achermann P, Trachsel L, Tobler I (1989) Sleep initiation and initial sleep intensity: interaction of homeostatic and circadian mechanisms. J Biol Rhythms 4:149-160.

Broughton RJ, Aguirre M (1987) Differences between REM and NREM sleepiness measures by event related potentials (P300, CNV), MSLT and subjective assessment in narcolepsy-cataplexy. Electroencephalogr Clin Neurophysiol 67:317-326.
Brown EN, Czeisler CA (1992) The statistical analysis of circadian phase and amplitude in constant routine core temperature data. J Biol Rhythms 7:177-202.

Brunter DP, Dijk DJ, Tobler I, Borbély AA (1990) Effect of partial sleep deprivation on sleep stages and EEG power spectra: evidence for non-REM and REM sleep homeostasis. Electroencephalogr Clin Neurophysiol 75:492-499.

Buijs RM, Kalsbeek A, Van der Woude TP, Van Heerikhuize JJ, Shinn S (1993) Suprachiasmatic nucleus lesion increases corticosterone secretion. Am J Physiol 264:R1186-R1 192.

Buzsaki G, Bickford RG, Ponomareff G, Thal LJ, Mandel R, Gage FH (1988) Nucleus basalis and thalamic control of neocortical activity in the freely moving rat. J Neurosci 8:4007-4026.

Campbell S, Zulley J (1989) Evidence for circadian influence on human slow wave slecp during daytime slecp cpisodes. Psychophysiology 26:580-585.

Campbell SS, Dawson D, Anderson MW (1993) Alleviation of sleep maintenance insomnia with timed exposure to bright light. J Am Geriatr Soc 41:829-836.

Carskadon MA, Dement WC (1975) Sleep studies on a 90-minute day. Electroencephalogr Clin Neurophysiol 39:145-155.

Carskadon MA, Dement WC (1979) Effect of total sleep loss on sleep tendency. Percept Motor Skills 48:495-506.

Carskadon MA, Dement WC (1980) Distribution of REM sleep on a 90-minute sleep-wake schedule. Sleep 2:309-317.

Cohen RA, Albers HE (1991) Disruption of human circadian and cognitive regulation following a discrete hypothalamic lesion: a case study. Neurology 41:726-729.

Czeisler CA (1978) Human circadian physiology: internal organization of temperature, sleep-wake and neuroendocrine rhythms monitored in an environment free of time cues. Ph.D. thesis, Stanford University.

Czeisler CA (1994) The effect of light on the human circadian pacemaker. In: Ciba Foundation symposium 183, Circadian clocks and their adjustment (Waterhouse JM ed), pp 254-302. Chichester: Wiley.

Czeisler CA, Weitzman ED, Moore-Ede MC, Zimmerman JC, Knauer RS (1980a) Human sleep: its duration and organization depend on its circadian phase. Science 210:1264-1267.

Czeisler CA, Zimmerman JC, Ronda JM, Moore-Ede MC, Weitzman ED (1980b) Timing of REM sleep is coupled to the circadian rhythm of body temperature in man. Sleep 2:329-346.

Czeisler CA, Brown EN, Ronda JM, Kronauer RE, Richardson GS, Freitag WO (1985) A clinical method to assess the endogenous circadian phase (ECP) of the deep circadian oscillator in man. Sleep Res 14:295.

Czeisler CA, Dumont M, Duffy JF, Steinberg JD, Richardson GS, Brown EN, Sánchez R, Ríos CD, Ronda JM (1992) Association of sleep-wake habits in older people with changes in output of circadian pacemaker. Lancet 340:933-936.

Daan S, Beersma DGM, Borbély AA (1984) Timing of human sleep: recovery process gated by a circadian pacemaker. $\Lambda \mathrm{m} \mathrm{J}$ Physiol 246 : R161-R178

Dantz B, Edgar DM, Dement WC (1994) Circadian rhythms in narcolepsy: studies on a 90 minute day. Electroencephalogr Clin Neurophysiol 90:24-35.

Dijk DJ, Beersma DGM (1989) Effects of SWS deprivation on subsequent EEG power density and spontaneous sleep duration. Electroencephalogr Clin Neurophysiol 72:312-320.

Dijk DJ, Czeisler CA (1994) Paradoxical timing of the circadian rhythm of sleep propensity serves the consolidation of sleep and wakefulness in humans. Neurosci Lett 166:63-68.

Dijk DJ, Beersma DGM, Daan S (1987a) EEG power density during nap sleep: reflection of an hourglass measuring the duration of prior wakefulness. J Biol Rhythms 2:207-219.

Dijk DJ, Visscher CA, Bloem GM, Beersma DGM, Daan S (1987b) Reduction of human sleep duration after bright light exposure in the morning. Neurosci Lett 73:181-186.

Dijk DJ, Beersma DGM, Daan S, Lewy AJ (1989) Bright morning light advances the human circadian system without affecting NREM sleep homeostasis. Am J Physiol 256:R106-R111.

Dijk DJ, Brunner DP, Beersma DGM, Borbély AA (1990a) EEG power density and slow wave sleep as a function of prior waking and circadian phase. Sleep 13:430-440.

Dijk DJ, Brunner DP, Borbély AA (1990b) Time course of EEG power density during long sleep in humans. Am J Physiol 258:R650-R661. 
Dijk DJ, Brunner DP, Borbély AA (1991) EEG power density during recovery sleep in the morning. Electroencephalogr Clin Neurophysiol 78:203-214

Dijk DJ, Duffy JF, Czeisler CA (1992) Circadian and sleep-wake dependent aspects of subjective alertness and cognitive performance. $\mathrm{J}$ Sleep Res 1:112-117

Dijk DJ, Hayes B, Czeisler CA (1993) Dynamics of electroencephalographic sleep spindles and slow wave activity in men: effect of sleep deprivation. Brain Res 626:190-199.

Dinges DF (1986) Differential effects of prior wakefulness and circadian phase on nap sleep. Electroencephalogr Clin Neurophysiol 64: 224-227.

Drennan M, Kripke DF, Gillin JC (1989) Bright light can delay human temperature rhythm independent of sleep. Am J Physiol 257:R136R141.

Eastman CI, Mistlberger RE, Rechtschaffen A (1984) Suprachiasmatic nuclei lesions eliminate circadian temperature and sleep rhythms in the rat. Physiol Behav 32:357-368.

Edgar DM (1986) Circadian time-keeping in the squirrel monkey: neural and photic control of sleep, brain temperature and drinking. Ph.D. thesis, University of California, Riverside.

Edgar DM, Dement WC, Fuller CA (1993) Effect of SCN-lesions on sleep in squirrel monkeys: evidence for opponent processes in sleepwake regulation. J Neuroscience 13:1065-1079.

Fuller CA, Lydic R, Sulzman FM, Albers HE, Tepper B, Moore-Ede MC (1981) Circadian rhythm of body temperature persists after suprachiasmatic lesions in the squirrel monkey. Am J Physiol 241: R385-R391

Fulton JF, Bailey $P$ (1929) Tumors in the region of the third ventricle: their diagnosis and relation to pathological sleep. J Nerv Ment Dis $69: 1-25,145-164,261-277$.

Gillberg M, Åkerstedt T (1982) Body temperature and sleep at different times of day. Sleep 5:378-388.

Gillberg M. Akerstedt T (1991) The dynamics of the first sleep cycle. Sleep 14:147-154.

Gillberg M, Anderzen I, Åkerstedt T (1991) Recovery within day-time sleep after slow wave sleep suppression. Electroencephalogr Clin Neurophysiol 78:267-273.

Glotzbach SF, Heller HC (1994) Temperature regulation. In: Principles and practice of sleep medicine (Kryger MH, Roth T, Dement WC, eds), pp 260-275. Philadelphia: Saunders.

Hume KI, Mills JN (1977) Rhythms of REM and slow-wave sleep in subjects living on abnormal time schedules. Waking Sleeping 1:291296.

Johnson MP, Duffy JF, Dijk DJ, Ronda JM, Dyal CM, Czeisler CA (1993) Short-term memory, alertness and performance: a reappraisal of their relationship to body temperature. J Sleep Res 1:24-29.

Klein DC, Moore RY, Reppert SM, eds (1991) Suprachiasmatic nucleus: the mind's clock. New York: Oxford UP

Klein T, Martens H, Dijk DJ, Kronauer R, Seely EW, Czeisler CA (1993) Circadian sleep regulation in the absence of light perception: chronic non-24-hour circadian rhythm sleep disorder in a blind man with a regular 24-h sleep-wake schedule. Sleep 16:333-343.

Kleitman N (1939) Sleep and wakefulness. Chicago: University of Chicago.

Klerman EB, Dijk DJ, Czeisler CA, Kronauer RE (1992) Simulations using self-selected light--dark cycles: "free-running" protocols in humans result in an apparent $\tau$ significantly longer than the intrinsic $\tau$. Abstr SRBR Amelia Island.

Kronauer RE (1990) A quantitative model for the effects of light on the amplitude and phase of the deep circadian pacemaker, based on human data. In: Sleep ' 90 (Horne J $\Lambda$, ed), pp 306-309. Bochum: Pontenagel.

Lavie P (1986) Ultrashort sleep-waking schedule. III. "Gates" and "forbidden zones" for sleep. Electroencephalogr Clin Neurophysiol 63:414-425.

Lavie P, Oksenberg A, Zomer J (1979) "It's time, you must wake up now." Percept Mot Skills 49:447-450.

Lee YS, Klerman EB, Czeisler CA, Kronauer RE (1992) Can ambulatory temperature data be used to assess endogenous circadian phase? Sleep Res 21:380.

McCarley RW (1990) REM sleep as a biological rhythm: the phenomenology and a structural and mathematical model. In: Brainstem control of wakefulness and sleep (Steriade M, McCarley RW, eds), pp 363-395. New York: Plenum.
McCarley RW, Massaquoi SG (1992) Neurobiological structure of the revised limit cycle reciprocal interaction model of REM cycle control. J Sleep Res 1:132-137.

McCormick DA (1992) Neurotransmitter action in the thalamus and cerebral cortex and their role in neuromodulation of thalamocortical activity. Prog Neurobiol 39:377-388.

Meijer JH, Rietveld WJ (1989) Neurophysiology of the suprachiasmatic circadian pacemaker in rodents. Physiol Rev 69:671-707.

Metherate R, Cox CL, Ashe JH (1992) Cellular bases of neocortical activation: modulation of neural oscillations by the nucleus basalis and endogenous acetylcholine. J Neurosci 12:4701-4711.

Mistlberger RE, Bergmann BM, Waldenar W, Rechtschaffen A (1983) Recovery sleep following sleep deprivation in intact and suprachiasmatic nuclei-lesioned rats. Sleep 6:217-233.

Moore RY, Eichler VB (1972) Loss of circadian adrenal corticosterone rhythm following suprachiasmatic nucleus lesions in the rat. Brain Res 42:201-206.

Moore RY, Klein DC (1974) Visual pathways and the central neural control of a circadian rhythm in pineal serotonin $N$-acetyltransferase activity. Brain Res 71:17-33.

Rainnie DG, Grunze HCR, McCarley RW, Greene RW (1994) Adenosine inhibition of mesopontine cholinergic neurons: implications for EEG arousal. Science 263:689-692.

Rechtschaffen A, Kales A (1968) A manual of standardized terminology, techniques and scoring system for sleep stages of human subjects. Bethesda, MD: U.S. Department of IIealth, Education and Welfare, Public Health Service.

Rusak B, Zucker I (1979) Neural regulation of circadian rhythms. Physiol Rev 59:449-526.

Satinoff E, Prosser R (1988) Suprachiasmatic nuclear lesions eliminate circadian rhythms of drinking and activity, but not of body temperature, in male rats. $\mathbf{J}$ Biol Rhythms 3:1-22.

Shanahan TL, Czeisler CA (1991a) Intrinsic period of the endogenous circadian rhythm of plasma melatonin is consistent with that of core body temperature during forced desynchrony in a 21-year-old-man. Sleep Res 20a:557.

Shanahan TL, Czeisler C $\Lambda$ (199lb) Light exposure induces equivalent phase shifts of the endogenous circadian rhythms of circulating plasma melatonin and core body temperature in men. J Clin Endocrinol Metab 73:227-235.

Snyder F (1966) Towards an evolutionary theory of dreaming. Am J Psychiatry 123:121-136.

Stephan FK, Zucker I (1972) Circadian rhythms in drinking behavior and locomotor activity of rats are eliminated by hypothalamic lesion. Proc Natl Acad Sci 69:1583-1586.

Steriade M, Datta S, Paré D, Oakson G, Curró Dossi R (1990) Neuronal activities in brain-stem cholinergic nuclei related to tonic activation processes in thalamocortical systems. J Neurosci 10:25412559.

Steriade M, Curró Dossi R, Nuñez A (1991) Network modulation of a slow intrinsic oscillation of cat thalamocortical neurons implicated in sleep delta wave: cortically induced synchronization and brainstem cholinergic suppression. J Neurosci 11:3200-3217.

Steriade M, McCormick DA, Sejnowski TJ (1993a) Thalamocortical oscillations in the sleeping and aroused brain. Science 262:679-685.

Steriade M, Nuñez A, Amzica F (1993b) A novel slow $(<1 \mathrm{~Hz}$ ) oscillation of neocortical neurons in vivo: depolarizing and hyperpolarizing components. J Neurosci 13:3252-3265.

Steriade M, Contreras D, Amzica F (1994) Synchronized sleep oscillations and their paroxysmal developments. Trends Neurosci 17:199208.

Strogatz SH, Kronauer RE, Czeisler CA (1986) Circadian regulation dominates homeostatic control of sleep length and prior wake length in humans. Sleep 9:353-364.

Strogatz SH, Kronauer RE, Czeisler CA (1987) Circadian pacemaker interferes with sleep onset at specific times each day: role in insomnia. Am J Physiol 253:R172-R 178.

Tobler I, Groos G, Borbély AA (1983) The effect of sleep deprivation on sleep in rats with suprachiasmatic lesions. Neurosci Lett 42:4954.

Trachsel L, Edgar DM, Seidel WF, Heller HC, Dement WC (1992) Sleep homeostasis in suprachiasmatic nuclei-lesioned rats: effects of sleep deprivation and triazolam administration. Brain Res 589:253261.

Uchida S, Maloney T, March JD, Azari R, Feinberg I (1991) Sigma 
$(12-15 \mathrm{~Hz})$ and delta $(0.3-3 \mathrm{~Hz})$ EEG oscillate reciprocally within NREM sleep. Brain Res Bull 27:93-96.

Watts AG (1991) The efferent projections of the suprachiasmatic nucleus: anatomical insights into the control of circadian rhythms. In: Suprachiasmatic nucleus: the mind's clock (Klein DC, Moore RY, Reppert SM, eds), pp 77-106. New York: Oxford UP.

Webb WB, Agnew HW (1971) Stage 4 sleep. Influence of time course variables. Science 174:1354-1356.

Webb WB, Agnew HW (1975) Sleep efficiency for sleep-wake cycles of varied length. Psychophysiology 12:637-641.

Wehr T (1992) A brain-warming function for REM sleep. Neurosci Biobehav Rev 16:379-397.

Weitzman ED, Nogeire C, Perlow M, Fukushima D, Sassin J, McGregor P, Gallagher TF, Hellman L (1974) Effects of a prolonged 3-hour sleep-wake cycle on sleep stages, plasma cortisol, growth hormone and body temperature in man. J Clin Endocrinol Metab 38:10181030 .
Weitzman ED, Czeisler CA, Zimmerman JC, Ronda JM (1980) Timing of REM and stages $3+4$ sleep during temporal isolation in man. Sleep 2:391-407.

Wever RA (1979) The circadian system of man. Berlin: Springer

Zulley J (1979) Der Einfluss von Zeitgebern auf den Schlaf des Menschen. Frankfurt: Fischer.

Zulley J (1980) Distribution of REM sleep in entrained 24 hour and free-running sleep-wake cycles. Sleep 2:377-389.

Zulley J (1990) Day and night sleep; the bedrest condition. In: Sleep '90 (Horne JA, ed), pp 319-323. Bochum: Pontenagel.

Zulley J, Wever R (1982) Interaction between the sleep-wake cycle and the rhythm of rectal temperature. In: Vertebrate circadian systems: structure and physiology (Aschoff J, Daan S, Groos G, eds), pp 253-261. Berlin: Springer.

Zulley J, Wever R, Aschoff J (1981) The dependence of onset and duration of sleep on the circadian rhythm of rectal temperature. Pfluegers Arch 391:314-318. 